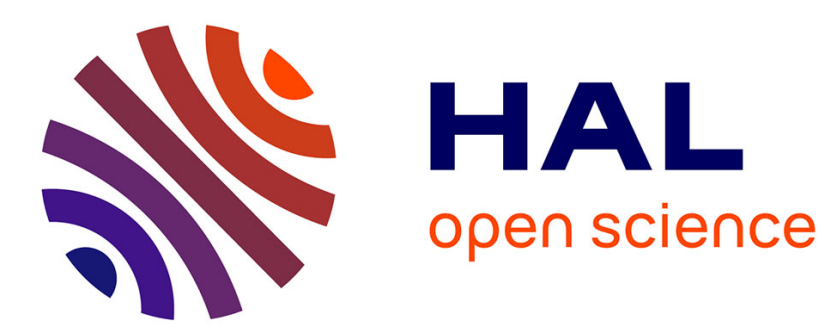

\title{
Revenus et systèmes de production. L'élevage bovin dans le Charolais
}

\author{
M. Bortzmeyer, J. Cavailhes, G. Lienard
}

\section{To cite this version:}

M. Bortzmeyer, J. Cavailhes, G. Lienard. Revenus et systèmes de production. L'élevage bovin dans le Charolais. Productions Animales, 1993, 6 (1), pp.79-81. hal-00896045

\section{HAL Id: hal-00896045 \\ https://hal.science/hal-00896045}

Submitted on 1 Jan 1993

HAL is a multi-disciplinary open access archive for the deposit and dissemination of scientific research documents, whether they are published or not. The documents may come from teaching and research institutions in France or abroad, or from public or private research centers.
L'archive ouverte pluridisciplinaire HAL, est destinée au dépôt et à la diffusion de documents scientifiques de niveau recherche, publiés ou non, émanant des établissements d'enseignement et de recherche français ou étrangers, des laboratoires publics ou privés. 
Cet article fait partie du dossier "Réforme de la PAC : simulations et analyses "publié par INRA Sciences sociales

\section{BORTZMEYER*, J. CAVAILHES** \\ G. LIENARD $* * * *$}

*INRA Dijon-OFIVAL,

**INRA Dijon,

***INRA Theix

\section{Revenus et systèmes de production. L'élevage bovin dans le Charolais}

La production de viande bovine est l'un des grands secteurs concernés par les décisions de réforme du 21 mai 1992 ; celle-ci prévoit une baisse de $15 \%$ du prix de la viande d'ici 1996, compensée par une forte revalorisation des primes à la vache allaitante et aux jeunes bovins mâles, dans la limite d'un niveau de chargement à l'hectare. L'effet de ces mesures variera selon les systèmes de production et les régions : les résultats de simulations micro-économiques d'exploitations-types par programmation linéaire présentés ici concernent la région charolaise.

\section{Des systèmes de production à cycle plus long}

L'aire charolaise centrale, qui s'étend sur les départements de l'Allier, de la Creuse, de la Nièvre et de la Saône-et-Loire, regroupe près de 800000 vaches nourrices $(23 \%$ du total national). Les systèmes de production, assez homogènes, sont extensifs, à base de prairies naturelles avec peu de consommations intermédiaires, sur des exploitations moyennes à grandes, produisant surtout des animaux "maigres" (à engraisser avant la vente en boucherie) et en particulier des broutards (âgés de 8 à 12 mois). La productivité du travail et les résultats économiques ont longtemps été comparables, voire supérieurs à ceux des autres régions productrices de viande bovine.

Lors de la simulation de "court terme", on observe une substitution du blé par l'orge et le développement des surfaces en maïs, accompagné d'un allongement du cycle d'élevage : la production de broutards lourds d'hiver (vendus à 12 mois environ), dominante dans la situation de référence, recule au profit de l'engraissement (taurillons de 18 ou 24 mois). Les exploitations qui ne peuvent produire de maïs augmentent leur sole céréalière.

La simulation est très sensible au rapport prix du maigre/prix du gras, largement indéterminé puisque l'accord du 21 mai fixe seulement un prix des animaux finis. Ainsi, par exemple, si le prix du maigre chute de $25 \%$, l'exploitation dirigée par un couple de niveau technique supérieur passe à l'engraissement de taurillons à l'auge, avec plus de céréales (sans prise en compte des investissements que cela peut impliquer), alors qu'on conserve un système à dominante de broutards si la baisse du prix n'est que de $15 \%$ pour le maigre et pour le gras (tableau 1).

Avec l'achat de terres, de cheptel et une augmentation de capital, ces tendances s'accentuent surtout chez les exploitants moins performants puisque les primes représentent une part plus importante de leur produit. Ce mouvement d'intensification simulé par le modèle va à l'encontre des évolutions des deux dernières décennies.

Ces résultats varient fortement suivant la présence ou l'absence de primes rattachées aux surfaces supplémentaires. Mais les productions sont, dans l'ensemble, peu sensibles au montant des primes, à l'exception des broutards d'automne de 8-9 mois, aujourd'hui très répandus et qui pourraient fortement régresser puisque la première prime aux mâles ne sera versée qu'à l'âge de dix mois.

\section{Vers un redressement du revenu}

Une simulation à échéance 1992, réalisée en intégrant les baisses de prix réelles (naturellement sans compensation), montre une baisse du résultat courant de $40 \%$, voire plus, par rapport à 1989, qui frappe très sévèrement les exploitants de niveau technique moyen. 
Tableau 1. Evolution des systèmes de production.

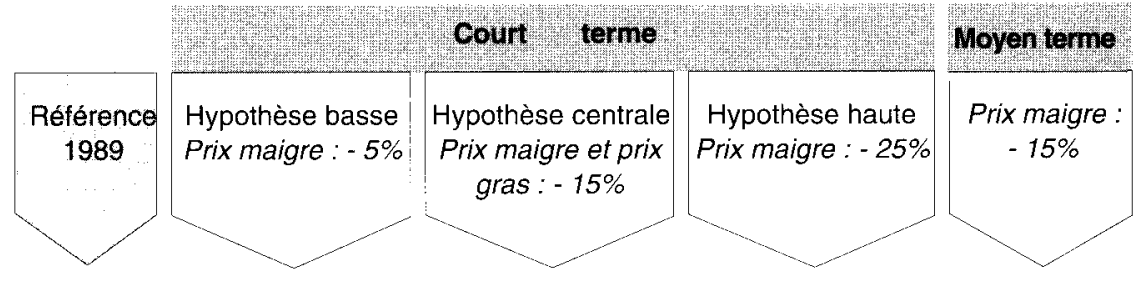

Exploitation conjugale

\section{performante}

SFP / SAU (\%)

Maïs (ha)

Système animal

100
1,2
broutards
lourds

99,8
1,2
broutards
lourds

99,1
1,2
broutards
lourds

87
3,8
taurillons
auge

6,3

taurillons

auge

\section{Exploitation conjugale \\ niveau technique moyen \\ SFP / SAU (\%) \\ Maïs (ha) \\ Système animal \\ dominant

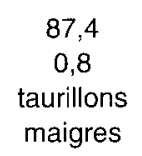 \\ 87,4 \\ taurillons \\ maigres}

\section{Méthodologie}

L'impact de la réforme est apprécié au niveau micro-économique, par programmation linéaire, ce qui permet de raisonner sous une hypothèse d'optimisation, dans le cadre des mesures connues (l'adaptation des marchés, en particulier la demande et le prix des animaux maigres, qui ne pourraient être simulés qu'au niveau macro-économique, sont largement inconnus).

Les activités et les paramètres sont fixés à dire d'experts, a partir de suivis de longue date de réseaux d'exploitations. On ne prend que les spéculations actuelles et, en respectant la cohérence des systèmes de production, on choisit un modèle simplifié comptetenu de l'imprécision du contexte des marchés. L'étalonnage sur l'année de référence (1989) permet de vérifier l'adéquation du modèle à la réalité.

Les simulations sont effectuées à "court terme", c'est-à-dire sans ajustement des facteurs fixes ni progrès technique, puis à "moyen terme", avec achat possible de facteurs quasi fixes et augmentation de la productivité du travail. L'horizon est le même dans les deux cas : 1996. La démarche strictement micro-économique oblige à fixer des quotas de primes individuels pour les céréales et les jeunes bovins.

Les cas simulés sont définis en fonction des dotations en facteurs fixes, des caractéristiques familiales et du niveau technique de manière à représenter au mieux la population (les quotas de primes donnent une place décisive au système de production antérieur à la réforme). On rend compte ici des cas suivants : un couple d'âge moyen, "en phase de croisière", sur 70 ha différencié par le niveau technique, bon ou moyen ; un chef jeune disposant de 60 ha et techniquement performant ; un exploitant pluri-actif sur une petite exploitation $(30 \mathrm{ha})$, suréquipé et de niveau technique moyen.
A l'horizon 1996, la simulation sans achat de terres permet un redressement du résultat par rapport à $1992:+35 \%$ pour les exploitations de bon niveau technique et +65 à $+85 \%$ pour les exploitations moins performantes qui bénéficient du caractère forfaitaire des aides. La baisse par rapport à 1989 est donc ramenée à $20 \%$ environ. Les aides représentent alors la quasi totalité du résultat, même pour les exploitations les plus performantes et $175 \%$ pour le cas d'un exploitant pluri-actif. Le revenu est done fortement déterminé par leur niveau.

Mais d'ici 1996, des adaptations peuvent intervenir : agrandissement, hausse de la productivité du travail ou de la technicité, qui améliorent les résultats par rapport au "court terme". L'évolution dépendra de l'adaptabilité des exploitations : les exploitants performants, qui disposent d'un potentiel de croissance à partir de capacités d'autofinancement importantes, maintiennent leurs résultats par rapport à 1989 s'ils obtiennent des droits supplémentaires pour que les animaux nouveaux soient "primés". Dans les cas de niveau technique moyen, le revenu 1989 ne peut être retrouvé même avec des augmentations de surface de $20 \%$, sauf si les gains de technicité permettent de se rapprocher du niveau technique supérieur. Enfin, les exploitants se retrouvant en situation financière difficile et à qui tout investissement est interdit, ne pourront que difficilement s'adapter aux nouvelles règles du jeu (figure 1). Notons cependant que les mesures propres à la France n'ont pas été retenues dans ces simulations : parmi elles, la prime à l'herbe $(300 \mathrm{~F} / \mathrm{ha}$ de $\mathrm{STH}$ en régime plein pour les exploitations extensives) est favorable à la zone charolaise où de nombreuses exploitations peuvent avoir un chargement inférieur à $1 \mathrm{UGB} / \mathrm{h}$.

Les simulations montrent donc que, loin de procéder à un nivellement, le nouveau cadre institutionnel pourrait conduire à une diversification des évolutions. Le mécanisme de gestion des références tracera bien entendu le cadre essentiel de ces évolutions. 


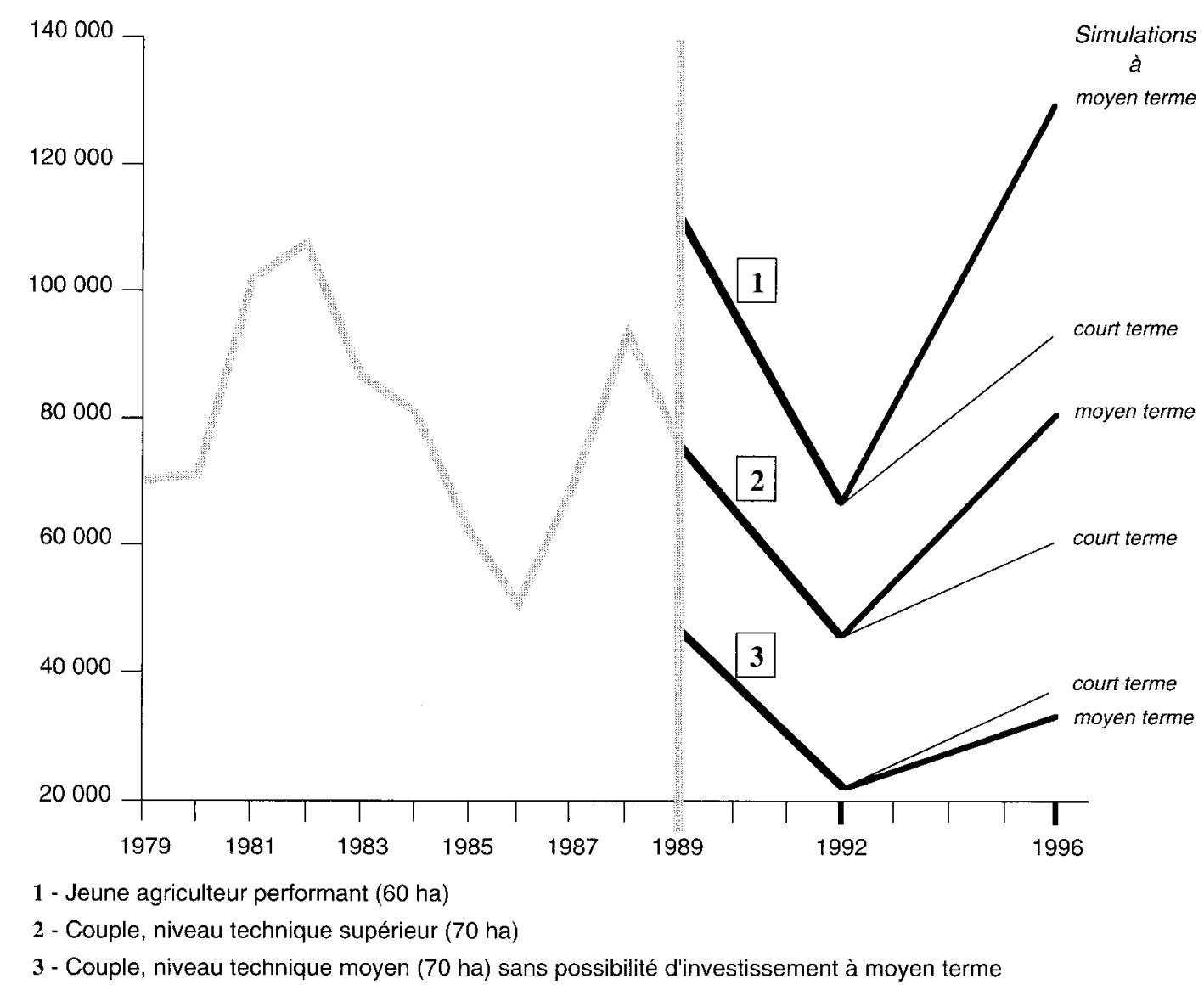

Figure 1. Evolution du résultat courant par travailleur (UTA) - 1979-1989: revenu courant avant impôt constaté.

\section{Pour en savoir plus :}

Bortzmeyer M., Impact de la réforme de la politique agricole commune sur les exploitations bovines de la zone charolaise, Dijon, Université de Bourgogne, Faculté de sciences économiques et de gestion, 1992, 95 p. + annexes (mémoire de DEA).
Cavailhès J., "L'économie charolaise. Évolution d'un système productif régional", Cahiers d'Economie et sociologie rurales, $\mathrm{n}^{\circ} 12,1989$, p. 8-54.

Lherm M., D. Bébin, G. Liénard, Systèmes de production charolais en Creuse. Références 1989, évolutions sur dix années : 1981-1990, Theix, INRAESR, 1992, $32 \mathrm{p}$. 